\title{
SÍNTESE E CARACTERIZAÇÃO DO ÓXIDO DE GRAFITE COMO PRECURSOR DE FOLHAS DE GRAFENO
}

\author{
C. B. $\operatorname{COSTA}^{1}$, D.P. da SILVA ${ }^{1}$, L.F. SENNA ${ }^{1}$, D.V. CESAR $^{1}$ \\ ${ }^{1}$ Universidade do Estado do Rio de Janeiro, Departamento de Química Analítica \\ E-mail para contato: dvargas@uerj.br
}

\begin{abstract}
RESUMO - O grafeno tem atraído considerável interesse por suas propriedades diferenciadas. Os processos de preparação envolvem diferentes metodologias, que se baseiam na produção do óxido de grafite $(\mathrm{GO})$ e sua posterior redução, com a formação do grafeno. O presente trabalho apresenta uma avaliação dos parâmetros de síntese do GO, com ênfase na relação molar grafite/oxidante e concentração de $\mathrm{H}_{2} \mathrm{O}_{2}$. A metodologia sugerida por Hummers, que se baseia na oxidação do grafite empregando uma mistura oxidante de nitrato de sódio e $\mathrm{H}_{2} \mathrm{SO}_{4}$, foi utilizada na obtenção do GO. Os resultados de difração de raios-X mostraram que as amostras preparadas apresentaram picos característicos do GO. As análises de espectroscopia de absorção no infravermelho confirmaram a presença dos grupos funcionais oxigenados $\mathrm{O}-\mathrm{H}, \mathrm{C}=\mathrm{O}$ e $\mathrm{C}-\mathrm{O}$, enquanto que a espectroscopia Raman permitiu identificar o grau de ordenamento e defeitos na estrutura. Os óxidos preparados apresentaram diferentes graus de espaçamento entre as folhas.
\end{abstract}

\section{INTRODUÇÃO}

O grafeno é um material bidimensional formado por átomos de carbono com uma estrutura cristalina hexagonal, cujas propriedades mecânicas e eletrônicas têm atraído interesse nas áreas tecnológica e de estudo fundamental. As propriedades físicas do grafeno são semelhantes àquelas dos nanotubos de carbono, porém possui uma superfície especifica maior $\left(\sim 2620 \mathrm{~m}^{2} / \mathrm{g}\right.$ - teórico) do que estes. No entanto, verifica-se que ocorrem dificuldades no preparo e obtenção de folhas isoladas, devido à dificuldade de esfoliação das folhas de grafeno, as quais tendem a formar aglomerados e retornar à forma de grafite (Hussain et al., 2006; Xu et al., 2008). Assim, a metodologia de preparação com elevado rendimento, controle da morfologia e formação de rugas e defeitos, constituem desafios que devem ser suplantados no processo de produção do grafeno.

De modo geral, diferentes métodos são usados para a esfoliação do grafite através de agentes oxidantes fortes e produção do óxido de grafite (GO), o qual é o precursor do grafeno e que posteriormente deverá sofrer um processo de redução para a formação do grafeno (Potts et al., 2011; Wang et al., 2013). A reação do grafite com os agentes oxidantes permite a introdução de grupos funcionais hidroxila e epóxido na rede cristalina, resultando em um aumento da distância interplanar do GO, bem como numa mudança na hibridação do $\mathrm{C}$ de $\mathrm{sp}^{2}$ para $\mathrm{sp}^{3}$ (Stankovich et al., 2006; Mastalir et al., 2008). O primeiro obstáculo no processo de obtenção do grafeno é sobrepor a elevada 


\section{9 a 22 de outubro de 2014 \\ Florianópolis/SC}

força de van der Waals que mantém a estrutura do grafite e, por consequência, as folhas de grafeno unidas. Dentre os métodos de preparação registrados na literatura, a metodologia proposta por Hummers (1958), seguida por diferentes modificações, constitui o processo predominantemente utilizado nos estudos envolvendo a obtenção do grafeno. Folhas de grafeno estáveis e com elevado rendimento (80\%) foram obtidas a partir da expansão térmica do $\mathrm{GO}$ em temperaturas superiores a $550{ }^{\circ} \mathrm{C}$ (McAllister et al., 2007). Geng, et al. (2009) propuseram uma nova rota que se baseia na esfoliação direta dos flocos de grafite em ácido fórmico e ultrassom, onde são apontadas como vantagens a elevada eficiência do processo de esfoliação, o uso de um intercalante não tóxico e a capacidade de produção em grande quantidade para fins industriais. Marcano et al. (2010) apresentaram uma modificação do método proposto por Hummers (1958) para a preparação do GO, onde o aumento da quantidade de $\mathrm{KMnO}_{4}$ e o uso de uma relação 9:1 da mistura $\mathrm{H}_{2} \mathrm{SO}_{4}: \mathrm{H}_{3} \mathrm{PO}_{4}$ aumentaram a eficiência do processo de oxidação. Recentemente, Yuan et al. (2011) utilizaram agentes redutores menos tóxicos como a glucose, que mostrou ser eficiente para o processo de obtenção do grafeno e na sua estabilidade.

Embora exista um grande número de trabalhos registrados na literatura, a preparação de folhas de grafeno permanece um desafio. As propriedades do grafeno mudam em função do número de folhas empilhadas e os agregados com mais de 10 folhas são considerados plaquetas finas de grafite (Potts et al., 2011). Portanto, o desenvolvimento de uma metodologia de preparação do grafeno com elevado rendimento e o controle dos parâmetros justificam a necessidade de estudos adicionais. $\mathrm{O}$ presente trabalho apresenta uma avaliação dos parâmetros de síntese do GO com ênfase na relação molar grafite/oxidante e concentração de peróxido de hidrogênio, com o objetivo de obter uma metodologia adequada à obtenção de um material estável e com elevado rendimento. As técnicas de microscopia eletrônica de varredura (SEM), difração de raios-X (XRD), espectroscopia de absorção na região do infravermelho (FTIR) e espectroscopia Raman (LRS) foram empregadas para a caracterização dos óxidos obtidos.

\section{MATERIAIS E MÉTODOS}

\subsection{Preparação do Óxido de Grafite}

O GO foi preparado a partir de grafite natural segundo a metodologia empregada por Hummers (1958). Primeiramente, o grafite em flocos foi tratado com solução de $\mathrm{HCl} 5 \%(\% \mathrm{~m} / \mathrm{v})$, seguido de filtração e lavagem com água destilada repetidas vezes. Ao final, foi seco em estufa a $100{ }^{\circ} \mathrm{C}$ por $12 \mathrm{~h}$. As etapas da síntese são descritas por Du et al. (2010). As relações de quantidades entre os reagentes utilizados na oxidação do grafite em função de cada preparação realizada são apresentadas na Tabela 1. Ao final, as amostras foram lavadas com água destilada até que o valor do pH da suspensão aquosa fosse igual a seis. As amostras, denominadas GOx (onde $\mathrm{x}=$ indica a síntese), foram armazenadas desta forma para posteriormente serem caracterizadas.

\subsection{Caracterizações}

Os óxidos obtidos foram caracterizados a partir das seguintes técnicas: 
Microscopia eletrônica de varredura por emissão de campo (FEG-SEM): usando um equipamento modelo Quanta 400 da FEI (30kV) e com microscópio da Carl Zeiss (EVO MA10) e sem recobrimento metálico.

Difração de raios-X (XRD): utilizando um difratômetro Rigaku D Max Ultima, com monocromador de grafite, radiação $\mathrm{Cu}-\mathrm{K} \alpha(\mathrm{V}=40 \mathrm{kV}$ e $\mathrm{I}=40 \mathrm{~mA})$. A analise foi realizada no intervalo de ângulo de Bragg (20) entre $5^{\circ}$ e $80^{\circ}$, com passo de $0,05^{\circ}$ e tempo de $2 \mathrm{~s}$. A identificação das fases formadas e determinação da distância interplanar $(d)$ foi realizada com o auxílio do programa MDI Jade 5 (Materials Data Inc), utilizando os arquivos de dados do International Center for Diffreaction Data ICDD PDF-2.

Espectroscopia de absorção na região do infravermelho médio (FTIR): realizadas em um espectrômetro Spectrum 100 (Perkin Elmer) com acessório de ATR, resolução de $4 \mathrm{~cm}^{-1}$ e detector DTGS.

Espectroscopia Raman (LRS): utilizando um espectrômetro HR-UV 800 (Horiba-Jobin Ivon), com detector CCD e microscópio confocal OLYMPUS BX 41, laser de He-Ne (632 nm).

Tabela 1 - Condições experimentais

\begin{tabular}{|c|c|c|c|}
\hline GO1 & GO2 & GO3 & GO4 \\
\hline $0,50 \mathrm{~g}$ de grafite & $0,10 \mathrm{~g}$ de grafite & $0,20 \mathrm{~g}$ de grafite & $0,20 \mathrm{~g}$ de grafite \\
\hline $0,25 \mathrm{~g} \mathrm{NaNO}_{3}$ & $0,25 \mathrm{~g} \mathrm{NaNO}_{3}$ & $0,25 \mathrm{~g} \mathrm{NaNO}_{3}$ & $0,25 \mathrm{~g} \mathrm{NaNO}_{3}$ \\
\hline $11,50 \mathrm{~mL}$ de $\mathrm{H}_{2} \mathrm{SO}_{4}$ & $11,50 \mathrm{~mL} \mathrm{H}_{2} \mathrm{SO}_{4}$ & $11,50 \mathrm{~mL} \mathrm{H}_{2} \mathrm{SO}_{4}$ & $11,50 \mathrm{~mL} \mathrm{H}_{2} \mathrm{SO}_{4}$ \\
\hline $1,5 \mathrm{~g} \mathrm{KMnO}_{4}$ & $1,5 \mathrm{~g} \mathrm{KMnO}_{4}$ & $1,5 \mathrm{~g} \mathrm{KMnO}_{4}$ & $1,5 \mathrm{~g} \mathrm{KMnO}_{4}$ \\
\hline $40 \mathrm{~mL}$ de $\mathrm{H}_{2} \mathrm{O}$ & $40 \mathrm{~mL}$ de $\mathrm{H}_{2} \mathrm{O}$ & $60 \mathrm{~mL}$ de $\mathrm{H}_{2} \mathrm{O}$ & $80 \mathrm{~mL}$ de $\mathrm{H}_{2} \mathrm{O}$ \\
\hline $40 \mathrm{~mL} \mathrm{H}_{2} \mathrm{O}_{2}(10 \%)$ & $60 \mathrm{~mL} \mathrm{H}_{2} \mathrm{O}_{2}(10 \%)$ & $60 \mathrm{~mL} \mathrm{H} \mathrm{O}_{2}(10 \%)$ & $60 \mathrm{~mL} \mathrm{H}_{2} \mathrm{O}_{2}(10 \%)$ \\
\hline
\end{tabular}

\section{RESULTADOS E DISCUSSÃO}

As análises de espectroscopia de absorção no infravermelho (FTIR) demonstraram que ocorreu a oxidação do grafite em todas as amostras, porém em diferentes graus. Os espectros de infravermelho, apresentados na Figura 1, apresentam bandas características de grupos funcionais oxigenados, como O-H, C-O e C=O (Stankovich et al., 2006; Geng et al., 2009; Yuan et al., 2011; Rattana et al., 2012). A comparação entre os espectros mostra diferenças no grau de oxidação das mesmas. Na amostra GO1 observam-se bandas de baixa intensidade relacionadas aos modos vibracionais das ligações $-\mathrm{OH}\left(3400 \mathrm{~cm}^{-1}\right), \mathrm{C}=\mathrm{O}\left(1730 \mathrm{~cm}^{-1}\right), \mathrm{C}-\mathrm{OH}\left(1228 \mathrm{~cm}^{-1}\right)$ e $\mathrm{C}-\mathrm{O}\left(1058 \mathrm{~cm}^{-1}\right)$. Para a amostra GO2 ocorreu um aumento significativo das bandas características dos modos vibracionais das ligações $-\mathrm{OH}, \mathrm{C}=\mathrm{O}$, e $\mathrm{C}-\mathrm{O}$, indicando maior grau de oxidação. Além disso, o surgimento das bandas em torno de $2900 \mathrm{~cm}^{-1}$, que se devem ao modo de estiramento da ligação C-H de grupos $\mathrm{CH}_{2}$ e $\mathrm{CH}_{3}$, sugerem uma modificação da estrutura pelo aumento de átomos de $\mathrm{C}$ com 
hibridação $\mathrm{sp}^{3}$. O mesmo comportamento foi observado para a amostra GO3. A amostra GO4 apresentou o mesmo perfil de bandas características dos grupos oxigenados e com maior intensidade, confirmando a ocorrência do processo de oxidação.

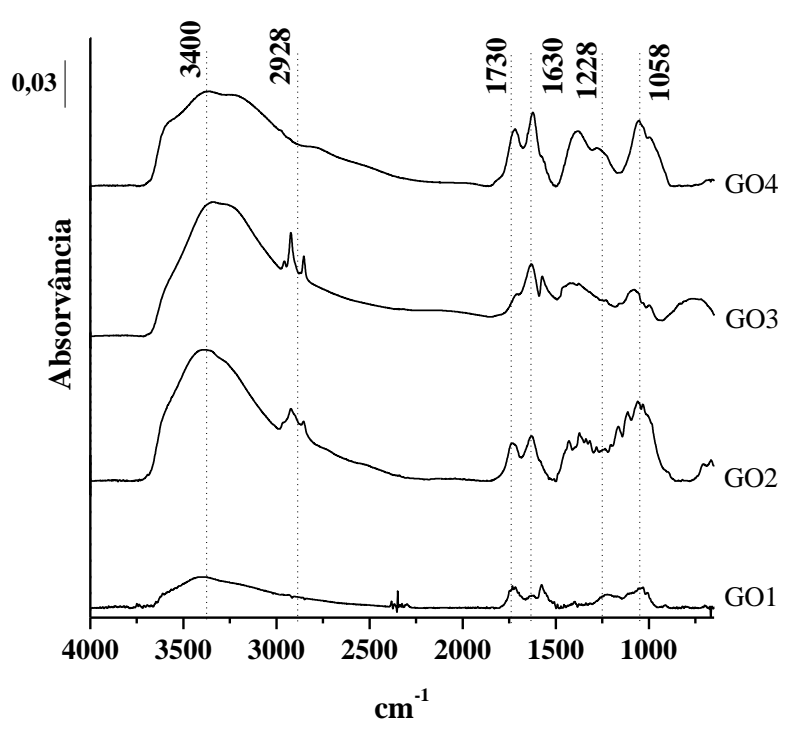

Figura 1 - Espectros de infravermelho das amostras sintetizadas.

Os resultados de difração de raios-X dos óxidos sintetizados são apresentados na Figura 2, juntamente com o padrão de difração do grafite usado como precursor dos óxidos.

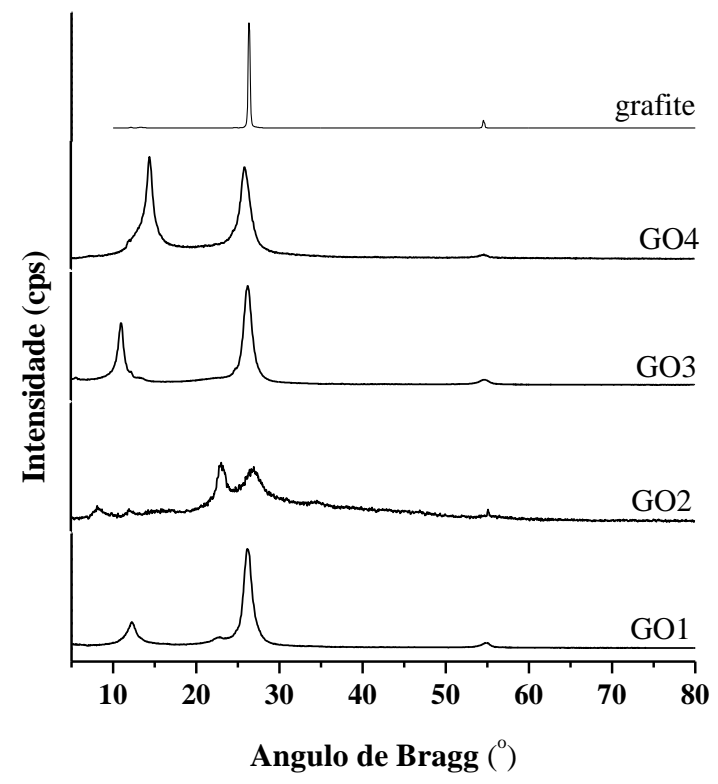

Figura 2 - Difratogramas de raios - $\mathrm{X}$ dos óxidos sintetizados e do grafite precursor. 
O grafite apresenta um pico de difração característico em $2 \theta=260\left(\mathrm{~d}_{002}=0,3375 \mathrm{~nm}\right.$, PDF 41-1487) e a diminuição de sua intensidade é utilizada para avaliar o processo de oxidação (Du et al., 2010; Zhao et al., 2014).

Nos óxidos sintetizados, o pico em $2 \theta=26^{\circ}$ ainda é observado, porém verifica-se um alargamento no mesmo, o que é indicativo de mudanças no ordenamento em relação ao material original. Além disso, os novos picos em $2 \theta<15^{\circ}$ confirmam as mudanças ocorridas na estrutura cristalina após o processo de oxidação e indicam um aumento da distancia interplanar (d) nestes óxidos (Geng et al., 2009; Zhao et al., 2014). Na amostra GO4, a presença de um pico em $2 \theta=14^{\circ}$ com intensidade ligeiramente superior ao pico em $2 \theta=26^{\circ}$ sugere maior eficiência no processo de oxidação do grafite.

Os espectros Raman (Figura 3) demonstram que todas as amostras apresentaram as bandas D (1330 a $1343 \mathrm{~cm}^{-1}$ ), que caracterizam os materiais com estruturas desordenadas e hibridação $\mathrm{sp}^{3}$, bem como a banda G (1596 - $\left.1607 \mathrm{~cm}^{-1}\right)$, associada a estruturas grafíticas com hibridação $\mathrm{sp}^{2}$. De acordo com a literatura, a banda D é atribuída ao defeito estrutural criado pela introdução de oxigênio contendo grupos funcionais na rede cristalina durante o processo de oxidação do grafite (Sivaram et al., 2004; Stankovich et al., 2007; Narksitipan et al., 2008; Yuan et al., 2011). Outro parâmetro associado aos resultados de Raman é a razão de intensidades das bandas D e G (ID/IG) das amostras, mesmo estas sofrendo modificações nos processos de oxidação. Por meio desse resultado foi possível identificar que as amostras possuem aproximadamente o mesmo grau de desordem estrutural, pois as razões ID/IG foram constantes para todas as amostras analisadas.

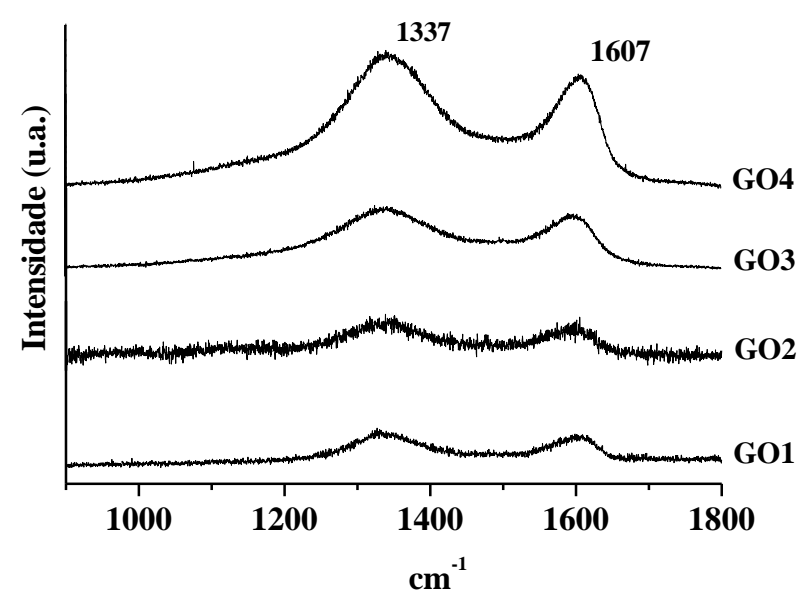

Figura 3 - Espectros Raman dos óxidos de grafite.

Para avaliar a morfologia dos óxidos sintetizados foram realizadas análises de microscopia eletrônica de varredura (Figuras 4a-e), que mostraram diferenças significativas entre os óxidos. A amostra GO1 (Figura 4a) apresentou espaçamento muito pequeno entre as folhas sugerindo baixo grau de oxidação, com morfologia semelhante a placas e similar ao grafite de partida (micrografia não apresentada). Este resultado é consistente com o observado por difração de raios-X e infravermelho. 

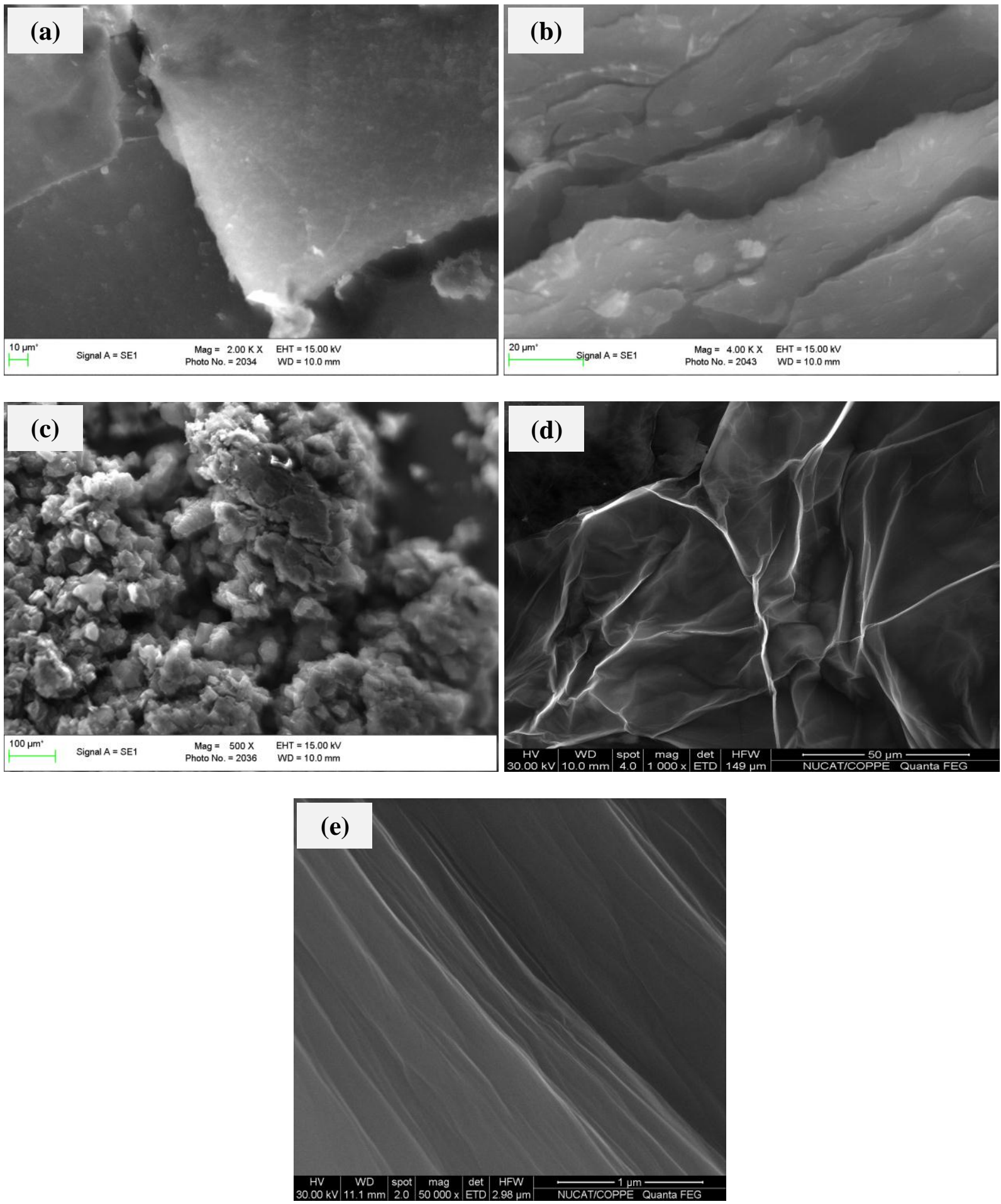

Figura 4 - Micrografias dos óxidos sintetizados: (a) GO1 (2000x); (b) GO2 (4000x); (c) GO3 (500x); (d) GO4 (1000x); (e) GO4 (50.000x). 


\section{9 a 22 de outubro de 2014 \\ Florianópolis/SC}

$\mathrm{Na}$ amostra GO2 (Figura 4b), as placas estão menores e com maior espaçamento, enquanto a amostra GO3 (Figura 4c) é formada por pequenos agregados, com perda da morfologia original do grafite. Estes resultados demonstram que o processo de oxidação ocorreu em diferentes graus em cada síntese, formou óxidos com baixo espaçamento interplanar e a morfologia de folhas ainda não foi obtida.

Por outro lado, a micrografia da amostra GO4 mostra que a morfologia é diferente dos demais óxidos, apresentando um aspecto de folhas de papel amassadas com rugas e dobras na superfície (Figura 4d), conforme observado em diversos estudos registrados na literatura (Stankovich et al., 2007; Geng et al., 2009; Du et al., 2010; Yuan et al., 2011). Na Figura 4e é possível observar as diversas folhas de óxido de grafite, cuja distancia interplanar $(d)$ obtida a partir dos resultados DRX foi de $0,6091 \mathrm{~nm}\left(2 \theta=14^{\circ}\right)$, que é aproximadamente o dobro do valor da distância entre os planos formadores do grafite $\left(\mathrm{d}_{002}=0,3375 \mathrm{~nm}\right)$. Isto se deve à introdução dos grupos oxigenados na estrutura (Yuan et al., 2011). Estes resultados demonstram que as condições utilizadas nesta preparação resultaram em maior eficiência do processo de oxidação e na formação de uma estrutura de folhas. No entanto, cabe observar que a total conversão do grafite em óxido não foi ainda obtida, conforme mostraram os resultados de difração de raios-X.

\section{CONCLUSÕES}

Os resultados obtidos no presente estudo permitiram verificar que óxidos de grafite são obtidos com diferentes graus de conversão do grafite, que depende da relação entre as quantidades de grafite, água e peróxido de hidrogênio. As análises de FTIR confirmaram a presença de grupos funcionais oxigenados $\mathrm{C}-\mathrm{O}, \mathrm{O}-\mathrm{H}, \mathrm{C}=\mathrm{O}$, evidenciando o processo de oxidação. A partir da espectroscopia Raman foi possível identificar o grau de ordenamento e defeitos na estrutura grafítica dos óxidos. O aumento da quantidade de peróxido de hidrogênio favorece o processo de oxidação do grafite, produzindo um óxido com morfologia de folhas de papel amassadas e com o dobro do espaçamento interplanar do grafite de partida. Embora a conversão total do grafite não tenha sido alcançada, os resultados são promissores e modificações no processo de síntese são necessárias para que seja obtido um elevado rendimento do óxido.

\section{AGRADECIMENTOS}

A Faperj pelo apoio financeiro.

Ao Instituto de Geologia (UERJ) pelas análises de SEM.

Ao Núcleo de Catálise/PEQ/COPPE/UFRJ pelas análises de Espectroscopia Raman e FEG-SEM.

\section{REFERÊNCIAS}

DU, Q.; ZHENG, M.; LIFENG, Z.; et al. Preparation of functionalized graphene sheets by a lowtemperature thermal exfoliation approach and their electrochemical supercapacitive behaviors. Electrochim. Acta, v. 55 , p. 3897-3903, 2010.

GENG, Y.; WANG,S.J.; KIM, J.K. Preparation of graphite nanoplatelets and graphene sheets. $J$. 
Colloid and Interf. Sci., v. 336, p. 592-598, 2009.

HUMMERS, W.; OFFEMAN, R. Preparation of Graphitic Oxide. J. Am. Chem. Soc., v. 80 (6), p.1339-1339, 1958.

HUSSAIN, F.; HOJJATI, M.; OKAMOTO, M., GORGA, R.E. Review article: Polymer-matrix Nanocomposites, Processing, Manufacturing, and Application: An Overview. J. Comp. Mater., v. 40, p. 1511, 2006.

MARCANO, D.C.; KOSYNKIN, D.V.; BERLIN, J.M.; SINITSKII, A.; SUN, Z.; SLESAREV, A.; et al. Improved Synthesis of Graphene Oxide. ACS Nano 4, p. 4806- 4814, 2010.

MASTALIR, A.; KIRÁLY, Z.; PATZKÓ, Á.; I. DÉKÁNY, P. L’ARGENTIERE. Synthesis and catalytic application of Pd nanoparticles in graphite oxide. Carbon, v. 46, p.1631, 2008.

MCALLISTER, M.J.; J-L.; LI, ADAMSON, D.H.; et al. Single Sheet Functionalized Graphene by Oxidation and Thermal Expansion of Graphite Chem. Mater., v. 19, p. 4396-4404, 2007.

NARKSITIPAN, S.; THONGTEM, T.; THONGTEM, S.; Characterization of sp3 carbon produced by plasma deposition on gamma-TiAl alloys. Appl. Surf. Science, v. 254, p. 7759-7764, 2008.

POTTS, J.R.; DREYER, D.R.; BIELAWSKI, C.W.; RUOFF, R.S. Graphene-based polymer nanocomposites. Polymer, v. 52, p. 5-25, 2011.

RATTANA, T.; et al. Preparation and characterization of graphene oxide nanosheets. Proc. Engineering. v. 32, p. $759-764,2012$.

SIVARAM, A.; NIKOLAEV, P.; GORELIK, O.; HADJIEV, V.G.; HOLMES, W.; FILES, B.; YOWELL, L. Protocol for the characterization of single-wall carbon nanotube material quality. Carbon 42, p. 1783-1791, 2004.

STANKOVIC, S.; DMITRIY, A.; DIKIN et al. Synthesis of graphene-based nanosheets via chemical reduction of exfoliated graphite oxide. Carbon , v. 45, p. 1558-1565, 2007.

STANKOVICH, S.; PINER, R.D.; NGUYEN, S.T.; RUOFF, R.S. Synthesis and exfoliation of isocyanate-treated graphene oxide nanoplatelets. Carbon, v. 44, p.3342-3347, 2006.

WANG, H., YUAN, X., WU, Y. et al. Graphene-based materials: Fabrication, characterization and application for the decontamination of wastewater and wastegas and hydrogen storage/generation. Adv. Colloid Inter. Science, v. 195-196, p. 19-40, 2013

XU, C.; WANG, X.; ZHU, J. Graphene-Metal Particle Nanocomposites. J. Phys. Chem. C, v. 112, p.19841-19845, 2008.

YUAN, W.; LI, B.; LI, L. A green synthetic approach to graphene nanosheets for hydrogen adsorption. Appl. Surf. Science, v. 257; p. 10183-10187, 2011.

ZHAO, W.; KIDO, G.; HARA, K.; NOGUCHI, H. Characterization of neutralized graphite oxide and its use in electric double layer capacitors. J. Electroanal. Chem. v 712, p. 185-193, 2014. 